\title{
VARIABILIDAD MORFOLÓGICA DE Phaseolus lunatus L. SILVESTRE DE LA REGIÓN OCCIDENTE DE MÉXICO
}

\section{MORPHOLOGICAL VARIABILITY OF WILD Phaseolus lunatus L. FROM THE WESTERN REGION OF MÉXICO}

\author{
José de J. López-Alcocer, Rogelio Lépiz-Ildefonso*, Diego R. González-Eguiarte, \\ Ramón Rodríguez-Macías y Eduardo López-Alcocer
}

\begin{abstract}
Centro Universitario de Ciencias Biológicas y Agropecuarias, Universidad de Guadalajara. Camino Ing. Ramón Padilla Sánchez, No. 2100. Las Agujas, Zapopan, Jalisco.

*Autor de correspondencia (rlepiz@cucba.udg.mx)
\end{abstract}

\section{RESUMEN}

Para conocer la variabilidad de poblaciones silvestres de Phaseolus lunatus L. originarias del occidente de México, se hizo la caracterización morfológica de 30 colectas. El experimento se estableció en Zapopan, Jalisco, México, en invernadero, en surcos de $4 \mathrm{~m}$ de longitud por población. La caracterización se efectuó en 10 plantas seleccionadas al azar con 25 descriptores cualitativos y 10 cuantitativos. A las variables cuantitativas se les calculó el coeficiente de correlación, análisis de agrupamiento y componentes principales. Las variables cualitativas color de hipocotilo, color de flores y color de vainas, presentaron diferencias significativas entre poblaciones; los rasgos cuantitativos de mayor variabilidad fueron: longitud y ancho de hoja primaria, longitud y ancho del foliolo central, días a floración, longitud de vaina y peso de 100 semillas. Hubo correlación positiva alta entre el peso de 100 semillas y longitud y ancho de hoja primaria, longitud de vaina y altitud de colecta. El análisis de conglomerados conformó dos grupos: el grupo A de floración tardía y el grupo B de floración precoz, y dentro de cada grupo se detectaron los subgrupos $\mathrm{A} 1$ y $\mathrm{B} 1$ de semillas pequeñas procedentes de localidades de baja altitud y los subgrupos A2 y B2 de semillas grandes originarios de localidades de mayor altitud. El análisis "Biplot" con los dos primeros componentes principales permitió separar a las poblaciones en tres grupos. El grupo $\mathrm{A}$ integrado por poblaciones de semillas grandes procedentes de zonas altas, el grupo B de semillas pequeñas originarias de zonas bajas, y el grupo $C$ intermedio y de mayor diversidad. Los resultados permiten un mejor entendimiento entre las poblaciones silvestres de frijol lima colectadas en el occidente de México.

Palabras clave: Phaseolus lunatus, conglomerados, componentes principales, frijol lima, variabilidad morfológica.

\section{SUMMARY}

With the aim of understanding the variability of wild populations of Phaseolus lunatus L. collected in Western México, the morphological characterization of 30 populations was carried out. The essay was established in a greenhouse at Zapopan, Jalisco, México, in rows of $4 \mathrm{~m}$ in length per population. For this characterization, 10 plants were randomly chosen per plot, on which 25 qualitative and 10 quantitative traits were recorded. Data from the quantitative variables were used to calculate coefficients of correlation, cluster analysis and principal components. The qualitative variables color of hypocotyl, color of flowers and color of pods, presented significant differences among populations; the quantitative characters of greater variability were length and width of the primary leaf, length and width of leaflet, days to flowering, pod length and weight of 100 seeds. High positive correlations were found between the eight of 100 seeds and length and width of primary leaf, pod length and altitude of the collection site. Cluster analysis formed two groups: group A of late flowering and group B of early flowering, and within each group two subgroups could be recognized: $A 1$ and $B 1$ of small seeds from the lowlands, and A2 and B2 of large seeds from the highlands. The Biplot analysis of the first two principal components separated the populations into three groups. Group A composed of large seeds from high places, group B of small seeds from the lower parts; and group C of greater diversity. These results allow for a better understanding of the relationships among wild populations of Phaseolus lunatus from Western México.

Index words: Phaseolus lunatus, conglomerates, principal components, lima bean, morphological variability.

\section{INTRODUCCIÓN}

Phaseolus lunatus L. conocido como frijol lima, Ib, comba, pallar, haba pallar o frijol mantequilla, es uno de los cinco taxa domesticados del género Phaseolus y es la segunda especie de mayor distribución, superficie cultivada y consumo del género Phaseolus en el mundo (GutiérrezSalgado et al., 1995; Delgado-Salinas et al., 1999). Se encuentra en áreas tropicales y subtropicales y se cultiva en varios países de América, así como en algunas regiones de Europa, Asia y África. Como todo frijol, constituye una rica fuente de proteínas, carbohidratos, hierro, calcio, fibra, y se distingue por tener bajo contenido de grasas (MartínezCastillo, 2015)

En el Continente Americano, Perú es el mayor productor de frijol lima, conocido como pallar, donde se siembran 7,000 ha y se cosechan $11,000 t$ anuales. Se cultiva principalmente en la costa peruana en el departamento de Ica, donde se produce una variedad de pallar grande blanco de exportación; por su tamaño, calidad y demanda internacional al departamento de lca se le ha concedido la denominación de origen de este pallar (Cabrera y Silva, 2008). El frijol lima también se cultiva y consume en Ecuador (tipo "big lima", conocido como haba pallar), Brasil (Silva do Carmo et al., 2013; Brito da Silva et al., 2015), Cuba (frijol caballero) (Castiñeiras et al., 2008) y Estados Unidos 
("big lima" y "baby lima", identificados como "butter bean") (USDA, 2012).

En México el frijol lima se siembra en la Península de Yucatán, en donde se le denomina lb o Ibe en Maya y representa el cuarto cultivo más importante integrado dentro del sistema de producción milpa (Martínez-Castillo et al., 2004). En esta región del país el ib domesticado presenta gran variación en la forma y color del grano. Se siembra en junio o julio asociado con maíz (Zea mays L.), sistema donde desarrolla lentamente; una vez que la gramínea llega a madurez y hay mayor penetración de luz en el sistema, el frijol muestra un desarrollo vigoroso, florece y produce. También se cultiva en la Depresión del Río Balsas, entre los estados de México, Guerrero y Michoacán, donde se le conoce como frijol comba y es muy aceptado por la población local; ahí se siembra en condiciones de secano o temporal, en pequeñas parcelas ubicadas en las vegas de ríos o laderas, asociado con maíz o en unicultivo con varas como tutor (Com. Pers.) ${ }^{1}$.

Con base en estudios morfológicos, bioquímicos y moleculares de la variabilidad genética efectuados principalmente con frijol silvestre, se han definido dos acervos genéticos de Phaseolus lunatus: Andino (A) y Mesoamericano (M) (Debouck et al., 1987; Gutiérrez-Salgado, 1995; Lioi, 1996; Fofana et al., 2001; Mota-Aldana et al., 2008; Serrano-Serrano et al., 2012). El acervo genético A de la forma domesticada se distingue por desarrollar órganos de mayor tamaño, especialmente semillas, las cuales son del tipo "big lima" o "baby lima", mientras que el complejo genético M, también de la forma domesticada, produce semilla más pequeña tipo "sieva" (Debouck et al., 1987).

En el acervo genético Mesoamericano que es diverso, tanto por su variabilidad genética como por su distribución geográfica, se han postulado dos grupos genéticos principales: Mesoamérica I (MI) y Mesoamérica II (MII) (SerranoSerrano et al., 2010, 2012), con una distribución geográfica distinta: MI, con presencia principal en el occidente de México, y Mll desde el sur de México y Centro América hasta el norte de Argentina (Andueza-Noh et al., 2013; SerranoSerrano et al., 2012). Adicionalmente, Martínez-Castillo et al. (2014), analizaron 67 poblaciones de P. lunatus silvestre originarias de México y mediante marcadores moleculares con microsatélites, y con base en su análisis proponen la existencia de dos subgrupos dentro de MI (Mla y Mlb) con sobre posición en su distribución geográfica. Lo anterior permite afirmar que la forma silvestre de $P$. lunatus presenta una amplia variabilidad genética en México, y se postula su ordenación en tres grupos genéticos Mla, Mlb y MII, cada uno a su vez con altos niveles de diversidad genética.

'Rogelio Lépiz, Fitomejorador, Centro Universitario de Ciencias Biológicas Agropecuarias, Universidad de Guadalajara.
Por lo que concierne a la domesticación de la especie, hasta ahora se han propuesto tres centros. Para el acervo genético Andino, el centro de domesticación se ubica en los valles entre Perú y Ecuador. Para el caso del acervo Mesoamericano, el proceso de domesticación habría ocurrido en dos áreas: la región occidente de México, entre los estados de Jalisco, Michoacán y Guerrero para el grupo genético Ml, y el área situada entre Guatemala y Costa Rica para el grupo genético MII (Andueza-Noh et al., 2013; Martínez-Castillo et al., 2014).

En cuanto a estudios específicos de caracterización morfológica en P. lunatus, Vargas et al. (2003) evaluaron 38 poblaciones silvestres de Costa Rica en las que encontraron amplia variación morfológica entre y dentro de las poblaciones, así como entre las regiones de origen; ellos sugieren la conservación de muchas poblaciones pequeñas, en lugar de un menor número de poblaciones grandes. En Cuba, Castiñeiras et al. (2008) evaluaron 30 variables y con análisis de conglomerados mediante distancias euclidianas en genotipos locales de P. lunatus, encontraron variabilidad entre los genotipos y definieron seis grupos morfo agronómicos del frijol caballero.

En México, Castillo-Mendoza et al. (2006) evaluaron con fines de conservación, la diversidad morfológica en 42 poblaciones domesticadas de P. coccineus (ayocote) y 107 de $P$. vulgaris originarias del oriente del Estado de México. Encontraron mayor variación en color de flor y semilla entre las poblaciones de frijol común, pero mayor diversidad en tamaño de semilla en frijol ayocote; asimismo detectaron dos grupos por precocidad, número de semillas por vaina y tamaño de semilla. Se determinó que todavía existe una gran diversidad en frijol común, mayor aun que en frijol ayocote, y que una forma de mantener la variabilidad in situ es el cultivo asociado de frijol con maíz por los campesinos.

Para entender las relaciones entre las formas cultivada, silvestre e intermedia de $P$. vulgaris en poblaciones colectadas en la región occidente de México, Lépiz et al. (2010) realizaron una caracterización morfológica en 15 poblaciones con base en 18 variables morfológicas cuantitativas. Los análisis de varianza, de agrupamiento con base en la distancia euclidiana y de componentes principales, precisaron la separación de las tres formas de frijol. Las poblaciones domesticadas formaron un grupo separado de las silvestres a una distancia de 2.08 unidades, mientras que las intermedias se ubicaron a una distancia de 1.23 unidades.

Meza-Vázquez et al. (2015) también caracterizaron morfológica y fenológicamente a 12 especies silvestres de Phaseolus y encontraron gran variabilidad en forma y 
tamaño de hoja primaria y foliolo central, longitud de vaina y semilla, así como del tamaño del polen y ciclo biológico. Las especies silvestres presentaron un comportamiento fenológico similar en las primeras etapas de desarrollo, pero en la fase reproductiva mostraron diferencias notables. Las especies de frijol se agruparon en dos conjuntos principales relacionados con el desarrollo de la planta y el ciclo biológico.

El potencial de los progenitores silvestres de frijol para ser usados en programas de mejoramiento genético, podrá rescatarse cuando se conozca la variabilidad genética de sus rasgos fenológicos, morfológicos, fisiológicos, bioquímicos y biofísicos asociados a la calidad agronómica, culinaria y nutricional (Peña-Valdivia et al., 2013). Se ha planteado que la reducida ganancia genética por mejoramiento genético del frijol común en los centros de investigación nacionales e internacionales, puede deberse a la estrecha base genética de la forma domesticada y a la introducción limitada de diversidad genética en las líneas élite de los programas de mejoramiento (Singh, 1989; Beebe et al., 1995).

La presente investigación se hizo con el objetivo de conocer la variabilidad morfológica en frijol silvestre de P. Iunatus oriundo de la región occidente de México, como una contribución al desarrollo de estrategias para su conservación y utilización en programas de mejoramiento genético.

\section{MATERIALES Y MÉTODOS}

La investigación se llevó a cabo en el año 2011, en Las Agujas, Municipio de Zapopan, Jalisco. El sitio presenta un clima semicálido subhúmedo con lluvias en verano (Medina et al., 1998), altitud de $1580 \mathrm{~m}$ y precipitación pluvial media anual de 979 mm (Ruiz-Corral et al., 2003). Se estudiaron 30 poblaciones silvestres de $P$. lunatus colectadas en los años 2003 a 2009 con el proyecto auspiciado por el Sistema Nacional de Recursos Fitogenéticos para la Alimentación y la Agricultura (SINAREFI) (Cuadro 1).

El experimento se estableció en invernadero; cada población se sembró en parcelas de $4 \mathrm{~m}$ de longitud y $1 \mathrm{~m}$ de ancho, distribuidas en serie sencilla. La semilla se escarificó con lija calibre 150, para acelerar y uniformar el proceso de germinación. Se sembraron 8 semillas por metro lineal de surco para dejar 16 plantas por parcela, de las cuales se seleccionaron 10 plantas al azar para la caracterización. Por el hábito trepador de los genotipos, fue necesario colocar espalderas de alambre recocido e hilo de rafia. Para cada surco, el alambre se amarró en los soportes metálicos laterales del invernadero a $1.80 \mathrm{~m}$ de altura, de donde se colgó un hilo de rafia que se amarró también en la base de la planta (un hilo por planta). Para asegurar un buen de- sarrollo de las poblaciones, se fertilizó a la siembra con el tratamiento 50-50-0 de $\mathrm{N}_{1} \mathrm{P}_{2} \mathrm{O}_{5}$ y $\mathrm{K}_{2} \mathrm{O}$, se regó dos veces por semana, y se controló a la maleza y a los insectos plaga (Trialeurodes vaporarorium, Westwood).

Para la caracterización se utilizó una guía de descriptores modificada, con base en los descriptores de frijol tanto del Centro Internacional de Agricultura Tropical (CIAT) como del Servicio Nacional de Inspección y Certificación de Semillas (SNICS-SAGARPA) (SNICS, 2005). Se registraron 25 variables cualitativas y 10 variables cuantitativas. Las variables morfológicas se registraron durante el desarrollo vegetativo y reproductivo de la planta; los descriptores de semilla se obtuvieron en postcosecha. Los datos de las variables cuantitativas se analizaron mediante promedios, desviaciones estándar, coeficientes de variación, análisis de correlación múltiple, de conglomerados y de componentes principales (CP) (Franco e Hidalgo, 2003; Ligarreto, 2003; Ligarreto y Martínez, 2002; Sánchez, 1995).

\section{RESULTADOS Y DISCUSIÓN}

\section{Caracterización morfológica}

Se observó amplia variación morfológica entre las poblaciones silvestres de $P$. lunatus originarias de la región occidente de México, tanto en variables cualitativas como cuantitativas. Todas las variables cualitativas mostraron variación, excepto en la forma del foliolo central y en la prominencia de las semillas en las vainas. El foliolo central en todas las poblaciones mostró forma acuminada, en tanto que la prominencia de las semillas en la vaina siempre se clasificó como ligera. Walma et al. (2007) también indicaron que todos los genotipos que estudiaron de esta especie, presentaron forma acuminada en los foliolos. La mitad de las poblaciones mostró hipocotilo verde y la otra mitad morado; 90 \% de las accesiones presentaron bractéolas pequeñas; $93 \%$ de los genotipos mostraron estandarte color verde; y $90 \%$ alas con tintes color lila; $80 \%$ tuvieron la sección transversal de la vaina de forma plana.

Con respecto al color de vaina, $53 \%$ de las accesiones mostraron color verde claro, $37 \%$ verde-amarillo y $10 \%$ verde-café; $50 \%$ mostraron apergaminado fuerte de vaina y $50 \%$ apergaminado medio. En la forma de semilla, 43 $\%$ presentó forma cuadrada, $23 \%$ elíptica, y $34 \%$ circular. En el aspecto de la testa en semilla, $67 \%$ fue brillante, 13 $\%$ intermedia y $20 \%$ opaca; respecto al color secundario predominante en la semilla, $47 \%$ presentaron color café y $53 \%$ color gris. La variación observada en foliolo central, color de la flor, tamaño de estípulas, forma de la vaina y forma de las semillas, es similar a la descrita para P. lunatus por Freytag y Debouck (2002) y Lépiz y Ramírez (2010). 
Cuadro 1. Procedencia y ubicación geográfica de las 30 poblaciones de Phaseolus lunatus.

\begin{tabular}{|c|c|c|c|c|}
\hline Clave del colector & Municipio & Latitud & Longitud & Altitud (m) \\
\hline ROL 167 & Zapopan, Jalisco & $20^{\circ} 44^{\prime}$ & $103^{\circ} 30^{\prime}$ & 1578 \\
\hline ROL 178 & Santiago Ixcuintla, Nayarit & $21^{\circ} 50^{\prime}$ & $105^{\circ} 08^{\prime}$ & 40 \\
\hline ROL 341 & Manzanillo, Colima & $19^{\circ} 05^{\prime}$ & $104^{\circ} 17^{\prime}$ & 16 \\
\hline ROL 348 & Tequila, Jalisco & $20^{\circ} 52^{\prime}$ & $103^{\circ} 50^{\prime}$ & 1319 \\
\hline ROL 359 & Tuxpan, Nayarit & $21^{\circ} 54^{\prime}$ & $105^{\circ} 15^{\prime}$ & 12 \\
\hline ROL 407 & Comala, Colima & $19^{\circ} 24^{\prime}$ & $103^{\circ} 38^{\prime}$ & 1475 \\
\hline ROL 428 & La Huerta, Jalisco & $19^{\circ} 19^{\prime}$ & $104^{\circ} 53^{\prime}$ & 10 \\
\hline ROL 448 & Santa María del Oro, Nayarit & $21^{\circ} 22^{\prime}$ & $104^{\circ} 34^{\prime}$ & 752 \\
\hline ROL 460 & Santa María del Oro, Nayarit & $21^{\circ} 21^{\prime}$ & $104^{\circ} 34^{\prime}$ & 791 \\
\hline ROL 464 & Tala, Jalisco & $20^{\circ} 39^{\prime}$ & $103^{\circ} 43^{\prime}$ & 1317 \\
\hline ROL 503 & Uruapan, Michoacán & $19^{\circ} 21^{\prime}$ & $102^{\circ} 04^{\prime}$ & 1446 \\
\hline ROL 506 & Lombardía, Michoacán & $19^{\circ} 13^{\prime}$ & $102^{\circ} 02^{\prime}$ & 889 \\
\hline ROL 511 & Villa Purificación, Jalisco & $19^{\circ} 21^{\prime}$ & $104^{\circ} 53^{\prime}$ & 40 \\
\hline ROL 513 & Casimiro Castillo, Jalisco & $19^{\circ} 36^{\prime}$ & $104^{\circ} 29^{\prime}$ & 311 \\
\hline ROL 528 & Villa Hidalgo, Nayarit & $21^{\circ} 47^{\prime}$ & $105^{\circ} 05^{\prime}$ & 81 \\
\hline ROL 535 & Mascota, Jalisco & $20^{\circ} 38^{\prime}$ & $104^{\circ} 51^{\prime}$ & 1503 \\
\hline ROL 542 & La Huerta, Jalisco & $19^{\circ} 28^{\prime}$ & $104^{\circ} 39^{\prime}$ & 388 \\
\hline ROL 543 & La Huerta, Jalisco & $19^{\circ} 24^{\prime}$ & $104^{\circ} 40^{\prime}$ & 391 \\
\hline ROL 544 & Cihuatlán, Jalisco & $19^{\circ} 18^{\prime}$ & $104^{\circ} 42^{\prime}$ & 119 \\
\hline ROL 546 & Cihuatlán, Jalisco & $19^{\circ} 13^{\prime}$ & $104^{\circ} 42^{\prime}$ & 9 \\
\hline ROL 547 & La Huerta, Jalisco & $19^{\circ} 35^{\prime}$ & $105^{\circ} 05^{\prime}$ & 21 \\
\hline ROL 549 & Tomatlán, Jalisco & $19^{\circ} 52^{\prime}$ & $105^{\circ} 19^{\prime}$ & 14 \\
\hline ROL 551 & El Tuito, Jalisco & $20^{\circ} 15^{\prime}$ & $105^{\circ} 18^{\prime}$ & 411 \\
\hline ROL 552 & Cabo Corrientes, Jalisco & $20^{\circ} 29^{\prime}$ & $105^{\circ} 17^{\prime}$ & 311 \\
\hline ROL 553 & Puerto Vallarta, Jalisco & $20^{\circ} 43^{\prime}$ & $105^{\circ} 11^{\prime}$ & 31 \\
\hline ROL 554 & San Sebastián del Oeste, Jalisco & $20^{\circ} 42^{\prime}$ & $104^{\circ} 53^{\prime}$ & 1539 \\
\hline ROL 555 & Zacualpan, Nayarit & $21^{\circ} 18^{\prime}$ & $105^{\circ} 09^{\prime}$ & 45 \\
\hline ROL 557 & El Llano, Nayarit & $21^{\circ} 25^{\prime}$ & $105^{\circ} 10^{\prime}$ & 37 \\
\hline ROL 559 & San Blas, Nayarit & $21^{\circ} 31^{\prime}$ & $105^{\circ} 13^{\prime}$ & 6 \\
\hline ROL 560 & Coahuayana, Michoacán & $18^{\circ} 35^{\prime}$ & $103^{\circ} 30^{\prime}$ & 184 \\
\hline
\end{tabular}

ROL = Rogelio Lépiz Ildefonso.

Todas las variables cuantitativas mostraron variabilidad genética. La anchura de vaina y número de semillas por vaina mostraron la menor variabilidad, en tanto que las dimensiones de la hoja primaria, los días a floración y el peso de la semilla, presentaron la mayor variabilidad (Cuadro 2). La variación en peso de 100 semillas fue desde 4.7 g en ROL 560 colectado en Coahuayana, Michoacán (184 msnm) hasta $21.9 \mathrm{~g}$ en ROL 535 oriundo de Mascota, Jalisco (1,503 msnm). Las características de mayor variabilidad fueron: longitud de hoja primaria, de $2.2 \mathrm{~cm}$ (ROL 553) a $8.6 \mathrm{~cm}$ (ROL535); anchura de hoja primaria, de $1.9 \mathrm{~cm}$ (ROL 553) a $7.9 \mathrm{~cm}$ (ROL 535); anchura de foliolo central, de $2.16 \mathrm{~cm}$ (ROL 167) a $5.16 \mathrm{~cm}$ (ROL 544), y longitud de foliolo central, de $3.78 \mathrm{~cm}$ (ROL 167) a $10.7 \mathrm{~cm}$ (ROL 554).
Por su parte el tiempo a floración mostró una diferencia de 31 d: desde 48 d (ROL 546 y ROL 552) hasta 79 días (ROL 448). Estos datos concuerdan en algunas características, con los descritos en P. lunatus por Lépiz y Ramírez (2010). En longitud de vaina se observó una variación de $2.5 \mathrm{~cm}$ (ROL 551) a $8.5 \mathrm{~cm}$ (ROL 535); al respecto, Vargas et al. (2003) encontraron que el promedio de esta variable fue de $4.0 \mathrm{~cm}$ en 30 poblaciones silvestres de Costa Rica,. El número de semillas por vaina varió de 1.3 (ROL 551) a 4.6 (ROL 553), datos similares a los reportados por Walma et al. (2007) quienes encontraron que el número de semillas por vaina en $P$. lunatus varió de 2 a 4.

El genotipo ROL 535 colectado en Mascota, Jalisco a 
Cuadro 2. Promedios de las variables cuantitativas registradas en la caracterización morfológica de 30 poblaciones silvestres de Phaseolus lunatus.

\begin{tabular}{|c|c|c|c|c|c|c|c|c|c|c|}
\hline Población & LHP & $\mathrm{AHP}$ & AFC & LFC & TF & LV & $\mathrm{AV}$ & NSV & LA & PCS \\
\hline ROL 167 & 5.0 & 4.6 & 2.16 & 3.78 & 68 & 4.9 & 1.6 & 2.4 & 2.3 & 13.6 \\
\hline ROL 178 & 4.5 & 4.1 & 2.38 & 3.92 & 49 & 4.5 & 1.7 & 2.3 & 2.5 & 6.3 \\
\hline ROL 341 & 3.4 & 3.0 & 3.55 & 6.23 & 59 & 4.2 & 1.2 & 1.7 & 2.6 & 6.4 \\
\hline ROL 348 & 4.8 & 4.4 & 2.86 & 4.06 & 64 & 5.2 & 1.4 & 3.1 & 2.3 & 11.4 \\
\hline ROL 359 & 3.5 & 3.3 & 2.76 & 5.16 & 64 & 5.0 & 1.4 & 2.3 & 2.5 & 7.4 \\
\hline ROL 407 & 6.4 & 4.9 & 2.75 & 5.10 & 69 & 6.0 & 1.7 & 3.6 & 3.1 & 17.6 \\
\hline ROL 428 & 3.4 & 3.1 & 2.95 & 5.05 & 68 & 4.6 & 1.0 & 3.8 & 2.0 & 8.2 \\
\hline ROL 448 & 4.2 & 3.5 & 2.60 & 4.02 & 79 & 5.2 & 1.3 & 2.6 & 3.7 & 9.2 \\
\hline ROL 460 & 4.9 & 4.0 & 2.82 & 4.76 & 59 & 4.0 & 1.2 & 1.6 & 3.7 & 9.6 \\
\hline ROL 464 & 6.7 & 5.9 & 4.32 & 6.96 & 53 & 5.3 & 1.4 & 1.5 & 3.1 & 11.9 \\
\hline ROL 503 & 7.8 & 6.2 & 3.58 & 8.86 & 51 & 6.7 & 1.7 & 1.8 & 2.4 & 17.6 \\
\hline ROL 506 & 4.4 & 3.8 & 5.00 & 8.18 & 53 & 5.0 & 1.4 & 2.6 & 2.9 & 7.3 \\
\hline ROL 511 & 4.6 & 3.8 & 4.00 & 6.18 & 53 & 3.6 & 1.2 & 2.2 & 2.0 & 6.8 \\
\hline ROL 513 & 4.4 & 3.9 & 3.84 & 5.52 & 53 & 4.2 & 1.2 & 1.9 & 3.0 & 8.4 \\
\hline ROL 528 & 6.0 & 4.7 & 4.34 & 8.68 & 66 & 4.3 & 1.3 & 2.5 & 3.5 & 8.0 \\
\hline ROL 535 & 8.6 & 7.9 & 3.80 & 7.26 & 49 & 8.5 & 2.0 & 1.8 & 5.2 & 21.9 \\
\hline ROL 542 & 4.7 & 4.1 & 4.00 & 7.52 & 53 & 4.0 & 1.0 & 1.7 & 2.2 & 7.2 \\
\hline ROL 543 & 5.2 & 4.1 & 4.12 & 7.62 & 58 & 4.0 & 1.1 & 3.0 & 1.6 & 7.3 \\
\hline ROL 544 & 4.9 & 4.2 & 5.18 & 8.56 & 53 & 4.5 & 1.0 & 2.6 & 2.0 & 5.6 \\
\hline ROL 546 & 5.8 & 4.7 & 4.34 & 6.78 & 48 & 5.2 & 1.2 & 2.2 & 2.6 & 7.4 \\
\hline ROL 547 & 5.2 & 4.4 & 4.02 & 6.48 & 53 & 4.0 & 1.2 & 2.7 & 2.7 & 6.8 \\
\hline ROL 549 & 4.7 & 4.2 & 2.67 & 4.95 & 58 & 4.0 & 4.2 & 1.8 & 1.9 & 6.7 \\
\hline ROL 551 & 5.0 & 4.4 & 3.70 & 6.24 & 68 & 2.5 & 1.0 & 1.3 & 1.7 & 6.4 \\
\hline ROL 552 & 4.0 & 3.0 & 4.00 & 6.44 & 48 & 3.3 & 1.1 & 1.6 & 1.4 & 5.2 \\
\hline ROL 553 & 2.2 & 1.9 & 3.00 & 3.80 & 68 & 3.0 & 0.8 & 4.6 & 2.5 & 4.8 \\
\hline ROL 554 & 7.4 & 6.4 & 4.60 & 10.70 & 63 & 5.1 & 1.5 & 1.6 & 3.7 & 16.0 \\
\hline ROL 555 & 4.3 & 3.3 & 3.18 & 5.14 & 63 & 4.5 & 1.0 & 2.0 & 1.1 & 6.2 \\
\hline ROL 557 & 4.2 & 3.9 & 3.14 & 6.68 & 53 & 4.0 & 1.1 & 1.9 & 3.3 & 5.6 \\
\hline ROL 559 & 3.7 & 3.5 & 2.48 & 4.08 & 58 & 3.9 & 1.1 & 3.6 & 1.2 & 8.2 \\
\hline ROL 560 & 3.9 & 3.4 & 2.86 & 4.36 & 53 & 4.2 & 1.2 & 1.6 & 1.7 & 4.7 \\
\hline Variación & $2.2-8.6$ & $1.9-7.9$ & $2.16-5.18$ & $3.78-10.7$ & $48-79$ & $2.5-8.5$ & $0.8-4.2$ & $1.3-4.6$ & $1.1-5.2$ & $4.7-21.9$ \\
\hline Promedio & 4.93 & 4.22 & 3.50 & 6.10 & 58.47 & 4.58 & 1.37 & 2.33 & 2.54 & 9.02 \\
\hline Des. est. & 1.38 & 1.17 & 0.80 & 1.78 & 7.83 & 1.13 & 0.60 & 0.92 & 0.88 & 4.34 \\
\hline CV (\%) & 28 & 28 & 23 & 29 & 13 & 25 & 44 & 39 & 35 & 48 \\
\hline
\end{tabular}

LHP = longitud de hoja primaria (cm); AHP = ancho de hoja primaria (cm); AFC = ancho del foliolo central (cm); LFC = longitud del foliolo central (cm); $\mathrm{TF}=$ tiempo a floración (d); LV= longitud de vaina $(\mathrm{cm}) ; A V=$ ancho de vaina (cm); NSV= número de semilla por vaina; LA = longitud de ápice (mm); PCS = peso de 100 semillas (g). Des. est. = desviación estándar; CV = coeficiente de variación.

1,503 m de altitud, registró los valores más altos en cinco de las diez variables cuantitativas: longitud y ancho de hoja primaria, longitud de vaina, longitud de ápice y peso de 100 semillas. En cambio, ROL 553 colectado en Puerto Vallarta, Jalisco a $31 \mathrm{~m}$ de altitud, mostró los valores más bajos en tres variables: longitud y ancho de hoja primaria y ancho de vaina, aunque mostró un alto número de semillas por vaina (Cuadro 2). Las variables morfológicas de mayores dimensiones en ROL 535 o menores en ROL 553 , están relacionadas con sus respectivos tamaños de semilla. Este mismo fenómeno se observa en variedades domesticadas de frijol común en las que los genotipos de semillas grandes desarrollan en general órganos de mayor tamaño (Kohashi-Shibata, 1991; White e Izquierdo, 1989). 
En el análisis de correlación múltiple entre las 10 variables cuantitativas más la variable altitud (Cuadro 3), se detectaron 17 correlaciones positivas mayores a 0.5 y seis superiores a 0.8. El peso de 100 semillas mostró correlación positiva alta con longitud de hoja primaria $(0.82 * *)$, ancho de hoja primaria $(0.83 * *)$, longitud de ápice $(0.59 *)$, longitud de vaina $(0.83 * *)$ y altitud del sitio de colecta $(0.86 * *)$. Lépiz et al. (2010) también encontraron valores de correlación semejantes entre algunas de las variables mencionadas al efectuar una caracterización morfológica en tres formas de frijol común.

Estos resultados sugieren que el tamaño de semilla en frijol lima silvestre está relacionado con el desarrollo y con las mayores dimensiones de algunos de sus órganos, como hojas primarias, foliolo central y vainas (KohashiShibata, 1991; White e Izquierdo, 1989). Además, la asociación positiva entre la altitud del sitio de colecta y las dimensiones mayores de hojas primarias, vainas, ápice y peso de semilla, permite postular que la altitud (donde prevalecen climas templados) favorece el desarrollo de órganos con mayores dimensiones, sin que esto signifique necesariamente un mayor ciclo biológico, puesto que la correlación entre días a floración y altitud de colecta fue baja (0.23).

\section{Análisis de conglomerados}

El análisis de conglomerados mediante el coeficiente de correlación practicado con 10 características morfológicas cuantitativas, separó las poblaciones de $P$. lunatus silvestre en dos grupos A y B (Figura 1). El grupo A integrado por 12 poblaciones, situado en la parte superior del dendrograma, es de floración tardía, entre 60 y 79 d después de la siembra. Dentro de este grupo nueve de ellas (subgrupo A1) son de semilla pequeña y fueron colectadas en las costas de Michoacán, Jalisco y Nayarit. El subgrupo A2 está integrado por genotipos tardíos de semillas grandes (ROL 167, ROL 348 y ROL 407), originarios de sitios con alturas mayores a 1,300 m. Dentro del grupo A destaca también la gran similitud entre ROL 428 y ROL 553 procedentes de la costa y de ROL 348 y ROL 407 originarias de localidades de altitud intermedia.

El grupo $\mathrm{B}$ integrado por 18 genotipos es más heterogéneo, de floración precoz entre 48 y $59 \mathrm{~d}$. El subgrupo B1 lo componen 14 poblaciones de semilla pequeña y oriundas de las costas de Michoacán, Colima, Jalisco y Nayarit. El subgrupo B2 con cuatro poblaciones de semilla grande, son originarias de sitios con altitudes mayores a 1,300 m; en este subgrupo está ROL 535 originario de Mascota, Jalisco con los granos más grandes (21.9 g en 100 semillas). En el grupo B destaca la gran similitud entre ROL 511 y ROL 552 y entre ROL 506 y ROL 544, todas ellas de semillas pequeñas y colectadas en sitios de baja altitud (Figura 1).

Por lo anterior se puede deducir que en la separación de los grupos A y B del dendrograma, la variable días a floración fue determinante. También es de destacar que los subgrupos A1 y B1 de semilla pequeña son oriundos de localidades de altitud menor que la de los sitios donde se colectaron los genotipos de los subgrupos A2 y B2 de grano grande. Este agrupamiento sin tomar en cuenta la precocidad, separa las poblaciones en dos grupos: genotipos de grano pequeño de partes bajas (A1 y B1) y genotipos

Cuadro 3. Coeficientes de correlación entre 10 variables cuantitativas registradas en la caracterización morfológica y altitud de origen, de 30 poblaciones silvestres de Phaseolus lunatus.

\begin{tabular}{|c|c|c|c|c|c|c|c|c|c|c|}
\hline & LHP & AHP & AFC & LFC & TF & LA & LV & AV & NSV & PCS \\
\hline \multicolumn{11}{|l|}{ LHP } \\
\hline AHP & $0.97 * *$ & & & & & & & & & \\
\hline AFC & 0.36 & 0.30 & & & & & & & & \\
\hline LFC & $0.59 *$ & $0.53^{\star}$ & $0.85 * *$ & & & & & & & \\
\hline DF & 0.01 & -0.01 & -0.32 & -0.17 & & & & & & \\
\hline $\mathrm{LA}$ & $0.53 *$ & $0.58 *$ & 0.13 & 0.26 & 0.06 & & & & & \\
\hline LV & 0.71 ** & $0.74 * *$ & 0.03 & 0.23 & 0.08 & $0.60 * *$ & & & & \\
\hline $\mathrm{AV}$ & 0.28 & 0.33 & -0.23 & -0.06 & -0.02 & 0.13 & 0.28 & & & \\
\hline SV & -0.36 & -0.38 & -0.25 & -0.35 & 0.37 & -0.14 & -0.06 & -0.20 & & \\
\hline PS & $0.82 \star \star$ & $0.83 * *$ & -0.02 & 0.26 & 0.32 & $0.59 *$ & $0.83 * *$ & 0.27 & -0.06 & \\
\hline A & $0.71 * *$ & $0.72 * *$ & 0.06 & 0.26 & 0.23 & 0.42 & $0.62 * *$ & 0.17 & -0.14 & $0.86 * *$ \\
\hline
\end{tabular}




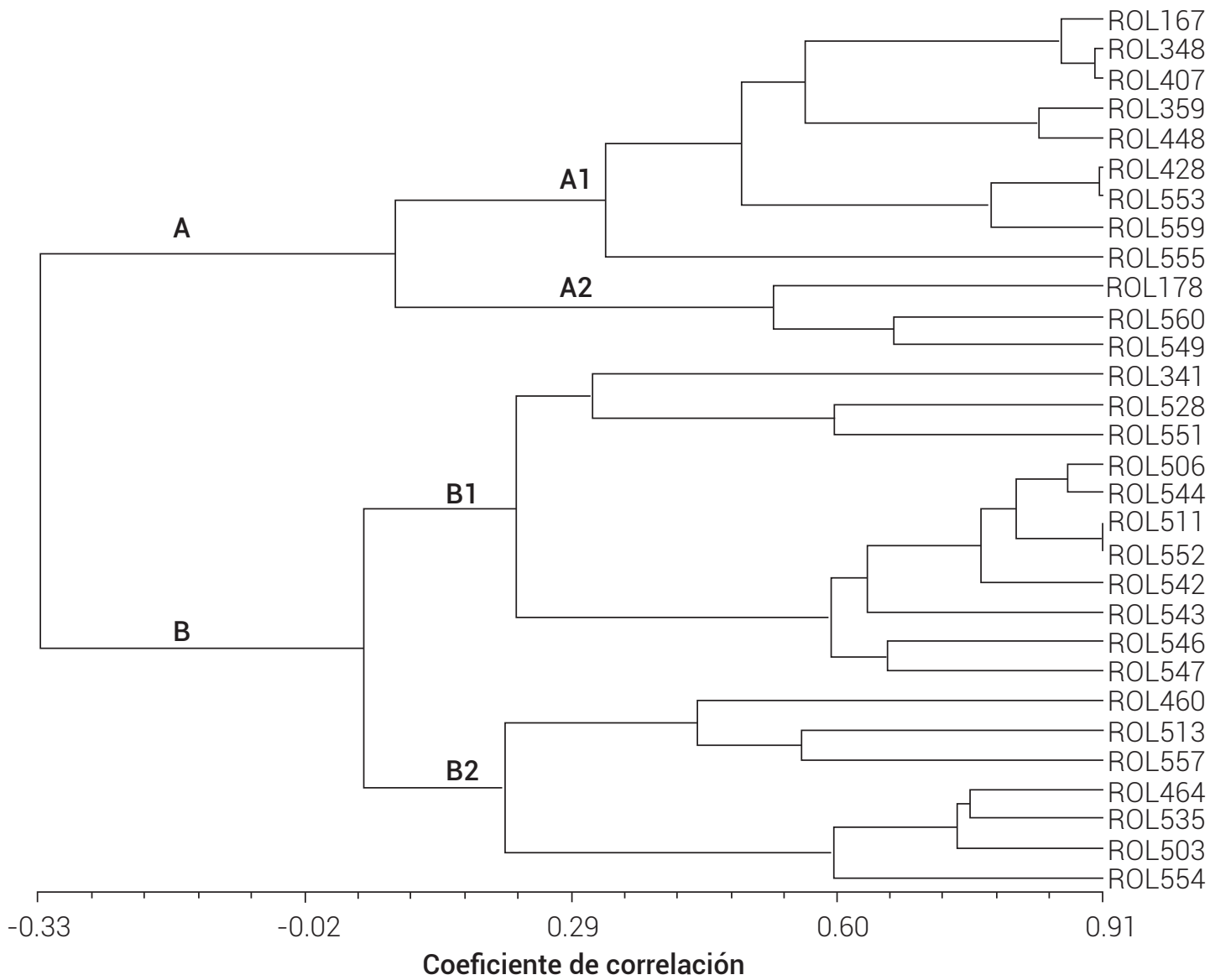

Figura 1. Dendrograma de 30 poblaciones silvestres de Phaseolus lunatus clasificadas con 10 variables morfológicas cuantitativas mediante el coeficiente de correlación.

de grano grande de partes altas (A2 y B2). Esta última separación de grupos por el tamaño de semilla y altitud del sitio de origen, podría estar asociada con los grupos M1a y M1b propuestos por Martínez et al. (2014), con sobre posición geográfica pero genéticamente diferentes entre sí, en cuyo caso los subgrupos A1 y B1 del presente estudio podrían ser del grupo M1a y los subgrupos A2 y B2 de M1b.

El análisis de componentes principales mostró que los primeros tres explicaron $78.61 \%$ de la variación total (Cuadro 4). El CP1 con $45.04 \%$ de la variación estuvo definido principalmente por las variables longitud y ancho de hoja primaria, longitud de vaina y peso de 100 semillas (Cuadro 5), variables que en el análisis de correlación múltiple presentan correlación positiva entre sí (Cuadro 3). El CP2, con una aportación a la varianza total de $21.42 \%$, se constituyó principalmente con las variables ancho y longitud de foliolo central (Cuadro 5), rasgos que mostraron asociación positiva en el análisis de correlación (Cuadro 3). Por su parte, el CP3 con una aportación a la varianza de $12.15 \%$, se integró por las variables tiempo a floración y número de semillas por vaina (Cuadro 3), con una correlación positiva intermedia entre ellas (0.37).

Los resultados del análisis "Biplot" (Figura 2) y los valores del $\mathrm{CP} 1$ con mayor contribución a la variabilidad total permitieron separar a las poblaciones en tres grupos. En el sector positivo y de mayor valor en la escala se ubicaron las poblaciones ROL 535, ROL 503, ROL 554, ROL 464 (grupo A); estos mismos genotipos se ubican en el subgrupo B2 del dendrograma y proceden de sitios con altitudes mayores a 1,300 $\mathrm{m}$. En el sector negativo y con menor valor en el CP1 están los genotipos ROL 553, ROL 559, ROL 428, ROL 560, ROL 555 (grupo B), mismos que se ubican en el subgrupo A1 del dendrograma, que provienen de sitios costeros de baja altitud. En la parte central de manera dispersa se constituyó un tercer conjunto (grupo C), en el que la mayoría de colectas tienen órganos de tamaño intermedio a pequeño, y son originarias de la parte costera con altitudes menores a los $500 \mathrm{~m}$, y pertenecen a los subgrupos A1, A2 y B1 del dendrograma.

Por la dirección y ángulo entre los vectores-variable que definen el grado de correlación entre sí, se observa un grupo 
Cuadro 4. Valores y porcentajes de la variación de cada componente de la matriz de 30 poblaciones silvestres de Phaseolus lunatus.

\begin{tabular}{lccc}
\hline Componente & Valor propio & \% de varianza & Varianza acumulada (\%) \\
\hline LHP & 4.50 & 45.04 & 45.04 \\
AHP & 2.14 & 21.42 & 66.46 \\
AFC & 1.21 & 12.15 & 78.61 \\
LFC & 0.64 & 6.44 & 85.05 \\
TF & 0.62 & 6.19 & 91.24 \\
LV & 0.49 & 4.90 & 96.14 \\
AV & 0.19 & 1.95 & 98.09 \\
NSV & 0.10 & 1.02 & 99.11 \\
LA & 0.07 & 0.66 & 99.77 \\
PCS & 0.02 & 0.23 & 100.00 \\
\hline
\end{tabular}

$\mathrm{LHP}=$ longitud de hoja primaria, $(\mathrm{cm})$ : AHP = ancho de hoja primaria $(\mathrm{cm}) ; \mathrm{AFC}=$ anchura del foliolo central (cm); LFC = longitud del foliolo central $(\mathrm{cm}) ; \mathrm{TF}=$ tiempo a floración (días); LV = longitud de vaina $(\mathrm{cm}) ; \mathrm{AV}=$ ancho de vaina $(\mathrm{cm}) ; \mathrm{NSV}=$ número de semillas por vaina; $\mathrm{LA}=$ longitud de ápice $(\mathrm{cm}) ; \mathrm{PCS}=$ peso de 100 semillas $(\mathrm{g})$.

Cuadro 5. Variables morfológicas de Phaseolus lunatus silvestre asociadas con los componentes principales más importantes.

\begin{tabular}{lccc}
\hline Variable morfológicas & CP1 & CP2 & CP3 \\
\hline Longitud de hoja primaria (cm) & 0.451 & -0.014 & 0.002 \\
Ancho de hoja primaria (cm) & 0.454 & -0.052 & -0.044 \\
Ancho del foliolo central (cm) & 0.191 & 0.545 & 0.280 \\
Longitud del foliolo central (cm) & 0.299 & 0.430 & 0.220 \\
Tiempo a floración (d) & -0.143 & -0.410 & 0.378 \\
Longitud de vaina (cm) & 0.374 & -0.271 & 0.067 \\
Ancho de vaina (cm) & 0.145 & -0.239 & -0.633 \\
Número de semillas por vaina & -0.191 & -0.287 & 0.509 \\
Longitud de ápice (mm) & 0.313 & -0.183 & 0.216 \\
Peso de 100 semillas (g) & 0.386 & -0.318 & 0.130 \\
\hline
\end{tabular}

del lado positivo del CP1 conformado por los vectores-variable longitud de hoja primaria (1), ancho de hoja primaria (2), longitud de vaina (6), longitud de ápice (9) y peso de 100 semillas (10) que tienen alta correlación entre ellas y con proximidad espacial a las poblaciones ROL 535, ROL 503, ROL 554, ROL 464. Un segundo grupo de vectoresvariable con nivel intermedio de correlación y también del lado positivo, lo integran ancho del foliolo central (3) y longitud del foliolo central (4), con proximidad a ROL 528 y ROL 506. Del lado negativo del CP1 se ubican los vectoresvariable días a floración (5) y número de semillas por vaina (8), cercanos espacialmente a las poblaciones ROL 178, ROL 359, ROL 428 y ROL 460.

En esta investigación los análisis de correlación, de conglomerados y del "Biplot" efectuados con los dos primeros componentes principales, muestran similitud en los resultados obtenidos. Esto muestra que las tres herramientas de análisis son complementarias y que en conjunto permiten un mejor entendimiento de las relaciones entre las poblaciones de P. lunatus L. y las variables morfológicas de orden cuantitativo involucradas.

\section{CONCLUSIÓN}

Las poblaciones silvestres de Phaseolus lunatus L. de la región occidente de México mostraron amplia variabilidad morfológica. Las variables cualitativas (color de hipocotilo, color de flor y de vaina, forma y color de la semilla), presentaron diferencias significativas. Las de orden cuantitativo con mayor variabilidad fueron longitud y ancho de hoja primaria, longitud y ancho del foliolo central, días a 


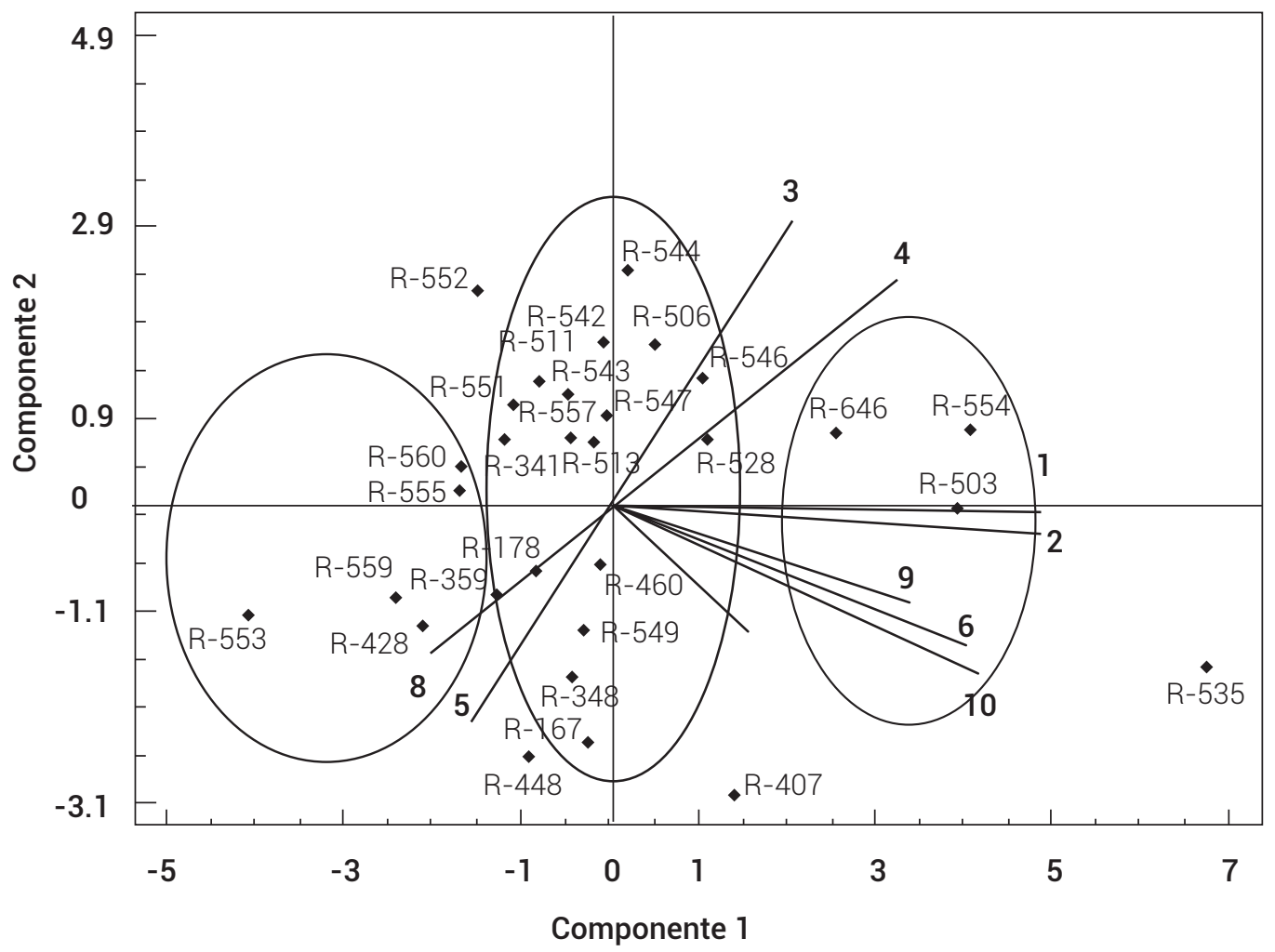

Figura 2. Ubicación de las 30 poblaciones silvestres y comportamiento de los vectores-variable con base en el análisis de componentes principales (CP) de 10 variables cuantitativas de Phaseolus lunatus. Claves de las variables: $1=$ longitud de hoja primaria $(\mathrm{cm}) ; 2=$ ancho hoja primaria $(\mathrm{cm}) ; 3=$ ancho del foliolo central $(\mathrm{cm}) ; 4=$ longitud del foliolo central $(\mathrm{cm})$; $5=$ tiempo a floración (días); 6 = longitud de vaina $(\mathrm{cm}) ; 7=$ ancho de vaina $(\mathrm{cm}) ; 8=$ número de semillas por vaina; $9=$ longitud de ápice $(\mathrm{mm}) ; 10=$ peso de 100 semillas $(\mathrm{g})$.

floración, longitud de vaina y peso de 100 semillas. Se encontró correlación positiva alta entre el peso de semilla y las variables longitud y ancho de hoja primaria, longitud de vaina y altitud de colecta. Los análisis de conglomerados y el "Biplot" elaborado con los dos primeros componentes principales detectaron dos grupos: uno integrado por poblaciones de semillas pequeñas originarias de sitios de colecta ubicados en bajas altitudes, y otro formado por accesiones de semillas grandes en sitios de mayor altitud.

\section{BIBLIOGRAFÍA}

Andueza-Noh R. H., M. L. Serrano-Serrano, M. I. Chacón-Sánchez, I. Sánchez del Pino, L. Camacho-Pérez, J. Coello-Coello, J. Mijangos C., D. G. Debouck and J. Martínez-Castillo (2013) Multiple domestications of the Mesoamerican gene pool of lima bean (Phaseolus lunatus L.): evidence from chloroplast DNA sequences. Genetic Resources and Crop Evolution 60:1069-1086.

Beebe S., P. Skroch, J. Thome, J. Niemhuis and J. Tivang (1995) Genetic diversity among common breeding lines developed for Central America. Crop Science 40:264-273.

Brito da Silva V., R. L. Ferreira, A. Celis de Almeida, C. T. Dos Santos and R. N. Oliveira (2015) Genetic diversity and promising crosses indication in lima bean (Phaseolus lunatus) accessions. Semina:
Ciências Agrárias 36:683-692.

Cabrera Vigil C. y J. Silva Guzmán (2008) Manual del Cultivo del Pallar. Fondo Empleo y CEDEP-Perú. Ica, Perú. $14 \mathrm{p}$.

Castillo-Mendoza M., P. Ramírez-Vallejo, F. Castillo-González y S MirandaColín (2006) Diversidad morfológica de poblaciones nativas de frijol común y frijol ayocote del oriente del Estado de México. Revista Fitotecnia Mexicana 29:111-119.

Castiñeiras L., L. Walón, N. León, T. Shagarodsky, O. Barrios, L. Fernández R. Cristóbal, Z. Fundora-Mayor, M. García, C. Giraudy, V. Fuentes, V. Moreno, F. Hernández, D. Arzola y D. de Armas (2008) Manejo de la variabilidad de Phaseolus lunatus (frijol caballero) conservada en comunidades rurales de Cuba. Revista del Jardín Botánico Nacional 29:151-160.

Debouck D. G., J. H. Lilian Jara, A. Campana Sierra and J. H. de la Cruz Rojas (1987) Observations on the domestication of Phaseolus lunatus L. Plant Genetic Resources Newsletters 70:26-32.

Delgado-Salinas A., T. Turley, A. Richman and M. Lavin (1999) Phylogenetic analysis of the cultivated and wild species of Phaseolus (Fabaceae). Systematic Botany 24:438-460

Fofana B., P. du Jardin and J. P. Baudoin (2001) Genetic diversity in the lima bean (Phaseolus lunatus L.) as revealed by chloroplast DNA (cpDNA) variations. Genetic Resources and Crop Evolution 15:02-05

Franco T. L. y R. Hidalgo (eds.) (2003) Análisis Estadístico de Datos de Caracterización Morfológica de Recursos Fitogenéticos. Boletín Técnico No. 8, Instituto Internacional de Recursos Fitogenéticos (IPGRI). Cali, Colombia. 89 p.

Freytag F. and D. G. Debouck (2002) Taxonomy, Distribution and Ecology 
of the Genus Phaseolus (Leguminoseae-papilionoideae) in North America, Mexico and Central America. SIDA, Botanical Miscellany. Botanical Research Institute of Texas, Fort Worth, TX, USA. $300 \mathrm{p}$

Gutierrez-Salgado A., P. Gepts and D. G. Debouck (1995) Evidence for two gene pools of the lima bean, Phaseolus lunatus $L$. in the Americas. Genetic Resources and Crop Evolution 2:15-28.

Kohashi-Shibata J. (1991) Fisiología. In: Contribuciones al Conocimiento del Frijol (Phaseolus) en México. E. M. Engleman (ed.). Colegio de Postgraduados. Chapingo, México. pp:39-58.

Lépiz I. R., J. J. López A., J. J. Sánchez G., F. Santacruz R., R. Romero N. y E. Rodríguez G. (2010) Características morfológicas de formas cultivadas, silvestres e intermedias de frijol común de hábito trepador. Revista Fitotecnia Mexicana 33:21-28.

Lépiz I. R. y R. Ramírez D. (2010) Los Parientes Silvestres del Frijol Común en el Occidente de México. Universidad de Guadalajara. México. 64 p.

Ligarreto M. G. A. (2003) Análisis de la variabilidad genética en fríjol. In: Análisis Estadístico de Datos de Caracterización Morfológica de Recursos Fitogenéticos. T. L. Franco y R. Hidalgo (eds.). Boletín Técnico No. 8, Instituto Internacional de Recursos Fitogenéticos (IPGRI), Cali, Colombia. pp:40-49.

Ligarreto M. G. A. y O. Martínez (2002) Variabilidad genética en frijol común (Phuseolus vulgaris L.). Análisis de variables morfológicas y agronómicas cuantitativas. Agronomía Colombiana 19:69-80.

Lioi L. (1996) Phaseolin diversity in wild lima beans (Phaseolus lunatus L.) in American Centers of Origen. Genetic Resources and Crop Evolution 43:575-580.

Martínez-Castillo J. (2015) Consideraciones sobre la importancia del muestreo en los estudios de la domesticación de plantas: el caso del frijol lima (Phaseolus lunatus) en México. Desde el Herbario CICY 7:17-22.

Martínez-Castillo J., L. Camacho-Pérez, S. Villanueva-Viramontes, R. H. Andueza-Noh and M. I. Chacon-Sánchez (2014) Genetic structure within the Mesoamerican Gene Pool of wild Phaseolus lunatus (Fabaceae) from Mexico as revealed by microsatellite markers: implications for conservation and the domestication of the species. American Journal of Botany 101:851-864.

Martínez-Castillo J., D. Zizumbo-Villarreal, H. Perales-Rivera and P. ColungaGarcía-Marín (2004) Intraspecific diversity and morpho-phenological variation in Phaseolus lunatus L. from the Yucatan Peninsula, Mexico. Economic Botany 58:354-380

Medina G. G., J. A. Ruiz C. y R. A. Martínez P. (1998) Los Climas de México. Centro de Investigación Regional del Pacífico Centro, INIFAP, SAGAR. México. $103 \mathrm{p}$

Meza-Vazquez K. E., R. Lépiz-Ildefonso, J. J. Lopez-Alcocer y M. M. MoralesRivera (2015) Caracterización morfológica y fenológica de es- pecies silvestres de frijol (Phaseolus). Revista Fitotecnia Mexicana 38:17-28.

Motta-Aldana J. R., M. L. Serrano-Serrano, J. Hernández-Torres, G. CastilloVillamizar, D. G. Debouck and M. I. Chacón (2008) Multiple origins of lima bean landraces in the Americas: Evidence from chloroplast and nuclear DNA polymorphisms. Crop Science 50:1773-1787.

Peña-Valdivia C. B., C. Trejo, C. Velázquez y A. López-Ordaz (2013) Reacción del frijol silvestre (Phaseolus vulgaris L.) a la profundidad de siembra. Revista Mexicana de Ciencias Agrícolas 4:89-102.

Ruiz-Corral J. A., I. J. González A., J. Anquiano C., I. Vizcaíno, D. Ibarra C., J. Alcalá G., V. Espinoza y H. E. Flores (2003) Estadísticas Climatológicas Básicas para el Estado de Jalisco, INIFAP-CIRPAC. México. $281 \mathrm{p}$

Sánchez G. J. J. (1995) El análisis Biplot en clasificación. Revista Fitotecnia Mexicana 18:188-203.

Serrano-Serrano M. L., R. H. Andueza-Noh, J. Martinez-Castillo, D. G. Debouck and M. I. Chacón (2012) Evolution and domestication of lima bean in Mexico: Evidence from ribosomal DNA. Crop Science 52:1698-1712.

Serrano-Serrano M. L., J. Hernández-Torres, G. Castillo-Villamizar, D. G. Debouck and M. I. Chacón (2010) Gene pools in wild lima bean (Phaseolus lunatus L.) from the Americas: Evidences for an Andean origin and past migrations. Molecular Phylogenetics and Evolution 54:76-87.

Silva do Carmo M. D., R. L. Ferreira G., A. C. de Almeida L., J. Souza da P., S. Oliveira G. and J. R. de Assunção F. (2013) Genetic variability in samples of determinate growth lima bean. Crop Breeding and Applied Biotechnology 13:158-164.

Singh S. P. (1989) Patterns of variation in cultivated common bean (Phaseolus vulgaris, Fabaceae). Economic Botany 43:39-57.

SNICS, Servicio Nacional de Inspección y Certificación de Semilla (2005) Guía Técnica para la Descripción Varietal de Frijol (Phaseolus vulgaris L.). SNIC/SAGARPA. Tlalnepantla, Estado de México. 22 p.

USDA, United States Department of Agriculture (2012) Vegetables and pulses: dry beans. Economic Research Service. http://www.ers. usda.gov/datafiles/Vegetable_and_Pulses_Yearbook_Tables.

Vargas E. M., E. Castro, G. Macaya y 0. J. Rocha (2003) Variación del tamaño de frutos y semillas en 38 poblaciones silvestres de Phaseolus lunatus (Fabaceae) del Valle Central de Costa Rica. Revista Biología Tropical 51:707-724

Walma N. R. G., L. S. Martins, E. F. da Silva, G. de M. G. Ferraz and F. J. de Oliveira (2007) Caracterização morfológica e molecular de acessos de feijão-fava (Phaseolus lunatus L.) Revista Brasileira de Engenharia Agrícola e Ambiental 11:37-45.

White J. W. y J. Izquierdo (1989) Frijol. Fisiología del Potencial de Rendimiento y la Tolerancia al Estrés. Boletín Técnico. CIAT, FAO. Santiago, Chile. 91 p. 